\title{
Modelling winter organic aerosol at the European scale with CAMx: evaluation and source apportionment with a VBS parameterization based on novel wood burning smog chamber experiments
}

Giancarlo Ciarelli et al.

Correspondence to: Sebnem Aksoyoglu (sebnem.aksoyoglu@psi.ch)

The copyright of individual parts of the supplement might differ from the CC BY 3.0 License. 


\section{Supplement}

Table S1. Statistics for model evaluation. $M_{i}$ represents the modelled value, $O_{i}$ the observations, $\bar{O}$ the mean of the observations and $n$ the total number of data points.

\begin{tabular}{|c|c|}
\hline Mean Bias (MB) & $M B=\frac{1}{n} \sum_{i=1}^{n}\left(M_{i}-O_{i}\right)$, \\
\hline Mean Error (ME) & $M E=\frac{1}{n} \sum_{i=1}^{n}\left(\left|M_{i}-O_{i}\right|\right)$, \\
\hline Mean Fractional Bias (MFB) & $M F B=\frac{2}{n} \sum_{i=1}^{n}\left(\frac{M_{i}-O_{i}}{M_{i}+O_{i}}\right)$, \\
\hline Mean Fractional Error (MFE) & $M F E=\frac{2}{n} \sum_{i=1}^{n}\left(\frac{M_{i}-O_{i} \mid}{M_{i}+O_{i}}\right)$, \\
\hline Coefficient of determination $\left(R^{2}\right)$ & $R^{2}=1-\frac{\sum_{i}^{n}\left(O_{i}-M_{i}\right)^{2}}{\sum_{i}^{n}\left(O_{i}-\bar{O}\right)^{2}}$. \\
\hline
\end{tabular}


Table S2. Statistical analysis for HOA during February-March 2009 periods at 11 AMS sites.

\begin{tabular}{|c|c|c|c|c|c|c|c|c|}
\hline Site & $\begin{array}{c}\text { Mean } \\
\text { observed } \\
\text { HOA } \\
\left(\mu \mathrm{g} \mathrm{m}^{-3}\right)\end{array}$ & $\begin{array}{c}\text { Mean } \\
\text { modelled } \\
\text { HOA } \\
\left(\mu \mathrm{g} \mathrm{m}^{-3}\right)\end{array}$ & $\begin{array}{c}\mathrm{MB} \\
\left(\mu \mathrm{g} \mathrm{m}^{-3}\right)\end{array}$ & $\begin{array}{c}\mathrm{ME} \\
\left(\mu \mathrm{g} \mathrm{m}^{-3}\right)\end{array}$ & $\begin{array}{c}\text { MFB } \\
{[-]}\end{array}$ & $\begin{array}{c}\text { MFE } \\
{[-]}\end{array}$ & $\mathrm{r}$ & $\mathrm{R}^{2}$ \\
\hline Barcelona & 2.1 & 0.8 & -1.3 & 1.5 & -0.4 & 0.8 & 0.4 & 0.1 \\
\hline Cabauw & 0.3 & 0.4 & 0.2 & 0.2 & 0.6 & 0.8 & 0.5 & 0.2 \\
\hline Chilbolton & 0.5 & 0.3 & -0.2 & 0.3 & -0.5 & 0.7 & 0.8 & 0.6 \\
\hline Helsinki & 0.4 & 1.0 & 0.6 & 0.7 & 0.8 & 0.9 & 0.2 & 0.1 \\
\hline Hyytiälä & 0.0 & 0.1 & 0.1 & 0.1 & 0.7 & 0.8 & 0.6 & 0.3 \\
\hline Mace Head & 0.1 & 0.1 & -0.1 & 0.1 & 0.5 & 1.1 & 0.6 & 0.3 \\
\hline Melpitz & 0.1 & 0.1 & 0.0 & 0.1 & -0.1 & 0.6 & 0.6 & 0.3 \\
\hline Montseny & 0.2 & 0.1 & -0.1 & 0.2 & -0.3 & 0.8 & 0.4 & 0.1 \\
\hline Payerne & 0.3 & 0.3 & -0.1 & 0.2 & 0.0 & 0.6 & 0.4 & 0.1 \\
\hline Puy de Dôme & 0.0 & 0.1 & 0.0 & 0.0 & 0.3 & 0.8 & 0.1 & 0.0 \\
\hline Vavihill & 0.4 & 0.2 & -0.2 & 0.2 & -0.4 & 0.7 & 0.5 & 0.2 \\
\hline
\end{tabular}


Table S3 Statistical analysis for BBPOA during February-March 2009 periods at 11 AMS sites.

\begin{tabular}{|c|c|c|c|c|c|c|c|c|}
\hline Site & $\begin{array}{c}\text { Mean } \\
\text { observed } \\
\text { BBPOA } \\
\left(\mu \mathrm{g} \mathrm{m}^{-3}\right)\end{array}$ & $\begin{array}{c}\text { Mean } \\
\text { modelled } \\
\text { BBPOA } \\
\left(\mu \mathrm{g} \mathrm{m}^{-3}\right)\end{array}$ & $\begin{array}{c}\mathrm{MB} \\
\left(\mu \mathrm{g} \mathrm{m}^{-3}\right)\end{array}$ & $\begin{array}{c}\mathrm{ME} \\
\left(\mu \mathrm{g} \mathrm{m}^{-3}\right)\end{array}$ & $\begin{array}{c}\text { MFB } \\
{[-]}\end{array}$ & $\begin{array}{c}\text { MFE } \\
{[-]}\end{array}$ & $\mathrm{r}$ & $\mathrm{R}^{2}$ \\
\hline Barcelona & 0.7 & 1.1 & 0.5 & 0.7 & 0.6 & 0.8 & 0.4 & 0.2 \\
\hline Cabauw & 0.1 & 0.5 & 0.3 & 0.3 & 1.0 & 1.1 & 0.5 & 0.3 \\
\hline Chilbolton & 0.5 & 0.2 & -0.3 & 0.3 & -0.6 & 0.8 & 0.6 & 0.4 \\
\hline Helsinki & 0.4 & 1.4 & 1.0 & 1.1 & 1.1 & 1.1 & 0.1 & 0.0 \\
\hline Hyytiälä & 0.1 & 0.5 & 0.4 & 0.4 & 1.5 & 1.5 & 0.7 & 0.5 \\
\hline Mace Head & 0.3 & 0.0 & -0.3 & 0.3 & -0.9 & 1.4 & -0.1 & 0.0 \\
\hline Melpitz & 0.2 & 0.3 & 0.1 & 0.2 & 0.7 & 0.9 & 0.4 & 0.2 \\
\hline Montseny & 0.2 & 0.3 & 0.1 & 0.2 & 0.5 & 0.8 & 0.2 & 0.1 \\
\hline Payerne & 0.4 & 0.8 & 0.4 & 0.5 & 0.8 & 0.9 & 0.6 & 0.3 \\
\hline Puy de Dôme & 0.1 & 0.2 & 0.1 & 0.2 & 0.5 & 1.0 & 0.3 & 0.1 \\
\hline Vavihill & 0.7 & 0.7 & 0.1 & 0.5 & -0.1 & 0.7 & 0.5 & 0.2 \\
\hline
\end{tabular}

Table S4. Comparison of statistics for BBPOA in VBS_BC_NEW with VBS_BC (average of all sites in February-March 2009)

\begin{tabular}{ccccccc}
\hline & $\begin{array}{c}\text { Mean obs } \\
\left(\mu \mathrm{g} \mathrm{m}^{-3}\right)\end{array}$ & $\begin{array}{c}\text { Mean mod } \\
\left(\mu \mathrm{g} \mathrm{m}^{-3}\right)\end{array}$ & $\begin{array}{c}\text { MB } \\
\left(\mu \mathrm{g} \mathrm{m}^{-3}\right)\end{array}$ & $\begin{array}{c}\text { ME } \\
\left(\mu \mathrm{g} \mathrm{m}^{-3}\right)\end{array}$ & $\begin{array}{c}\text { MFB } \\
{[-]}\end{array}$ & $\begin{array}{c}\text { MFE } \\
{[-]}\end{array}$ \\
\hline VBS_BC & 0.36 & 0.60 & 0.24 & 0.45 & 0.47 & 0.98 \\
VBS_BC_NEW & 0.36 & 0.59 & 0.23 & 0.43 & 0.50 & 0.97 \\
\hline
\end{tabular}



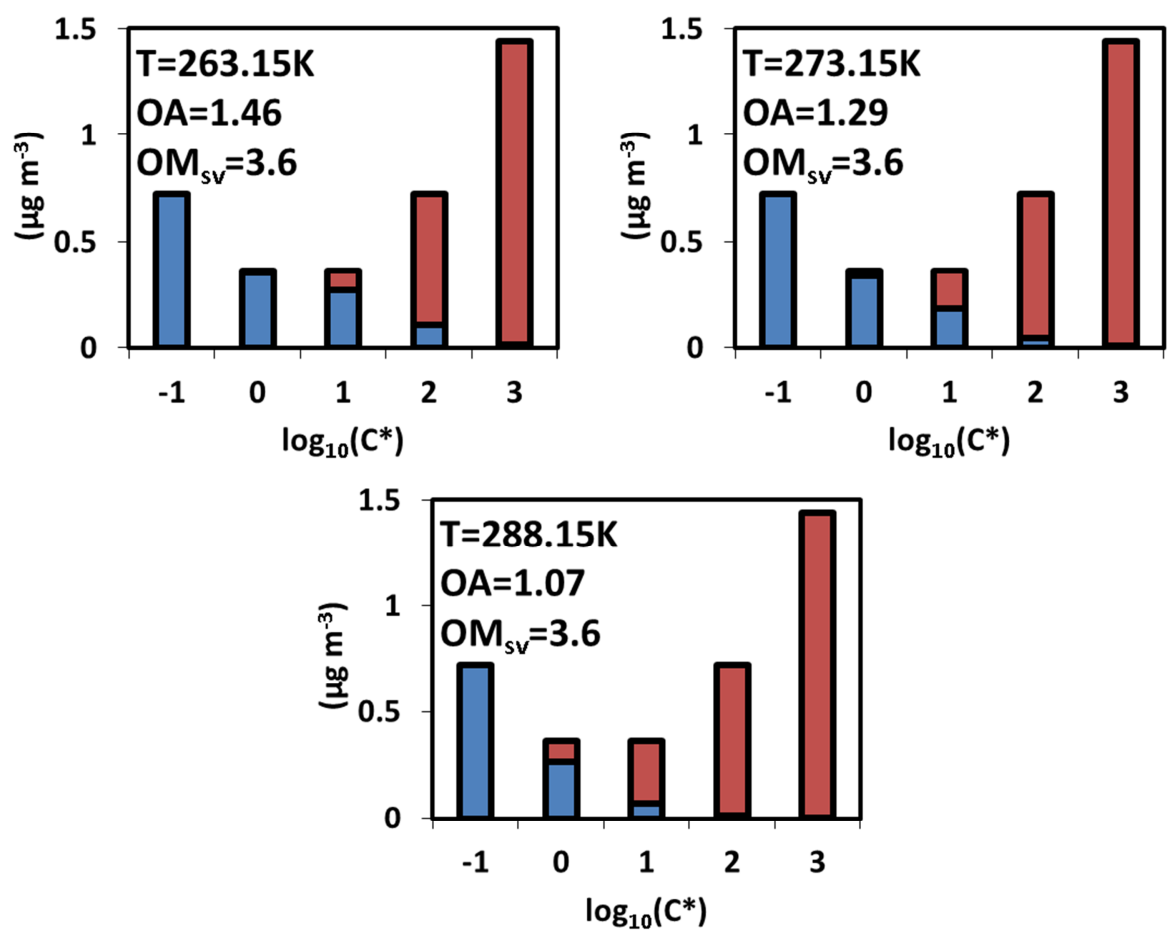

Figure S1. Box-model partitioning of biomass burning POA at about $1 \mu \mathrm{g} \mathrm{m} \mathrm{m}^{-3} \mathrm{OA}$ at different temperatures $(263.15,273.15$ and $288.15 \mathrm{~K})$ using volatility distributions proposed by May et al. (2013). Particle phase is represented blue and gas phase in red. The lowest bin $\left(\log _{10} \mathrm{C}^{*}=-1\right)$ is used as a proxy for all non-volatile species which will only reside in the particle phase. 

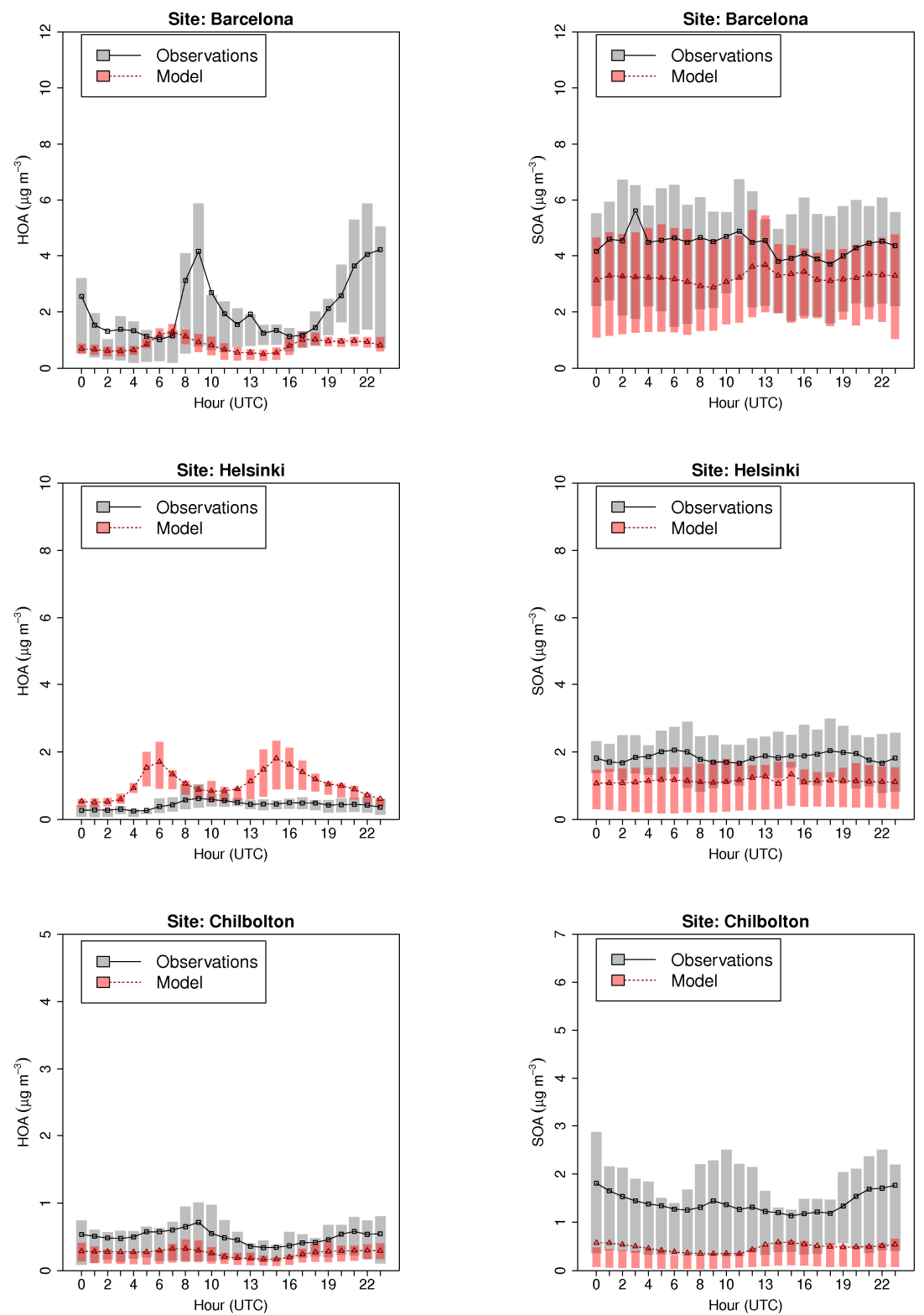

Figure S2. Comparison of modelled (red) and measured (grey) HOA and SOA diurnal profiles at the sites of Barcelona, Helsinki and Chilbolton. The extent of the bars indicates the 25th and 75th percentiles. 

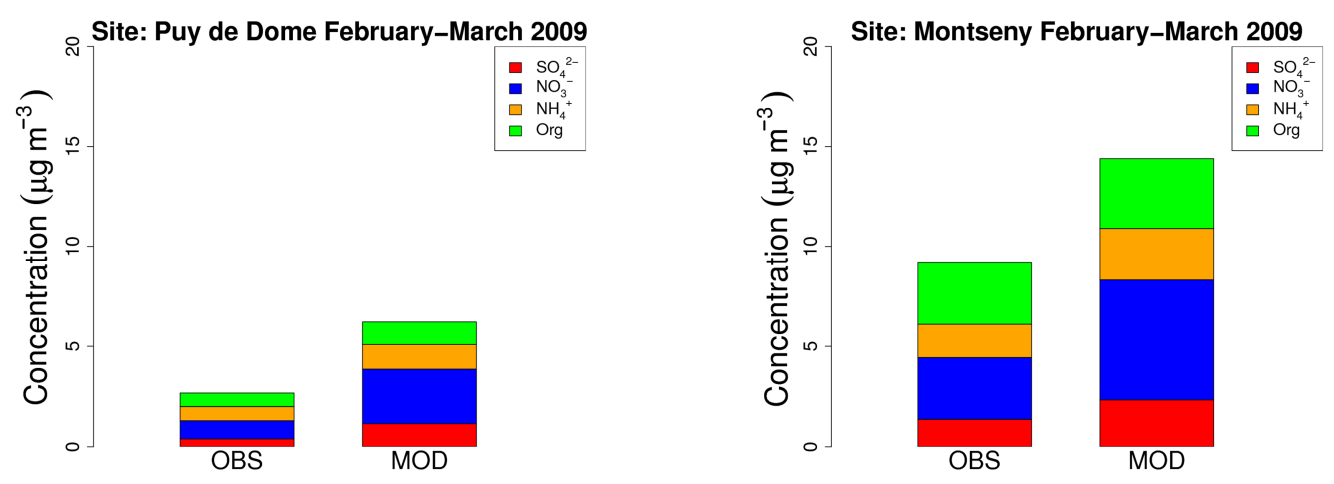

Figure S3. Comparison of modelled non-refractory PM25 components at Puy de Dome and Montseny with the AMS measurements in February-March 2009.
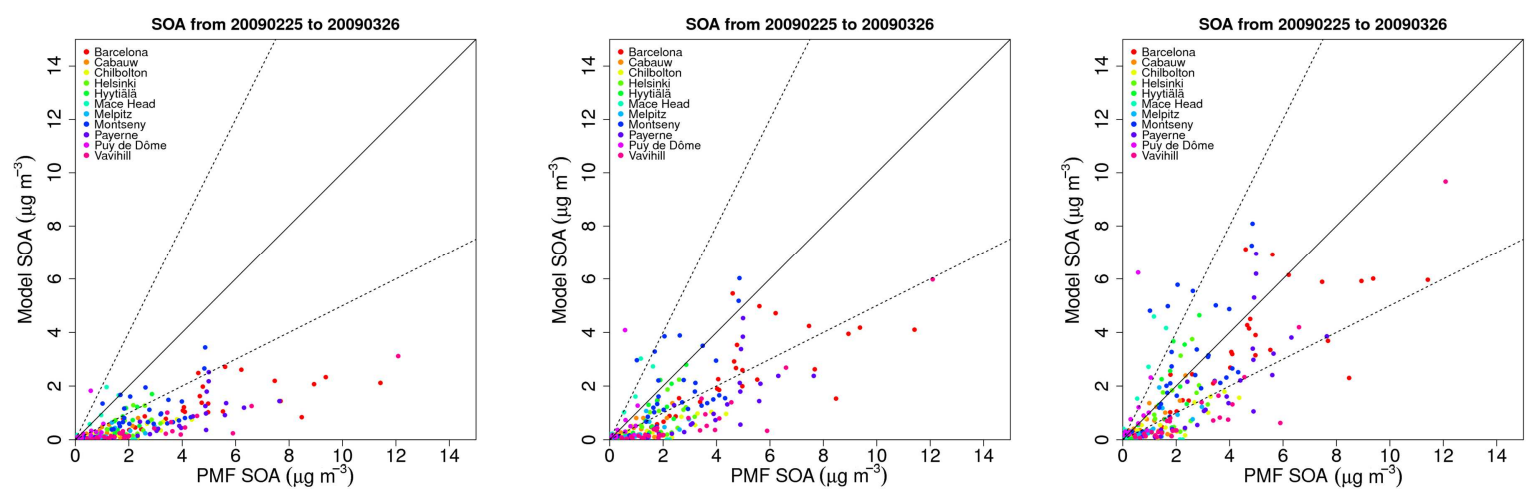

Figure S4. Modelled versus PMF SOA; with VBS_BC (Ciarelli et al., 2016) (left panel), with VBS_BC where BBPOA vapours were allowed to be further oxidized (Koo et al. 2014) (middle panel), and with VBS_BC_NEW (right panel).

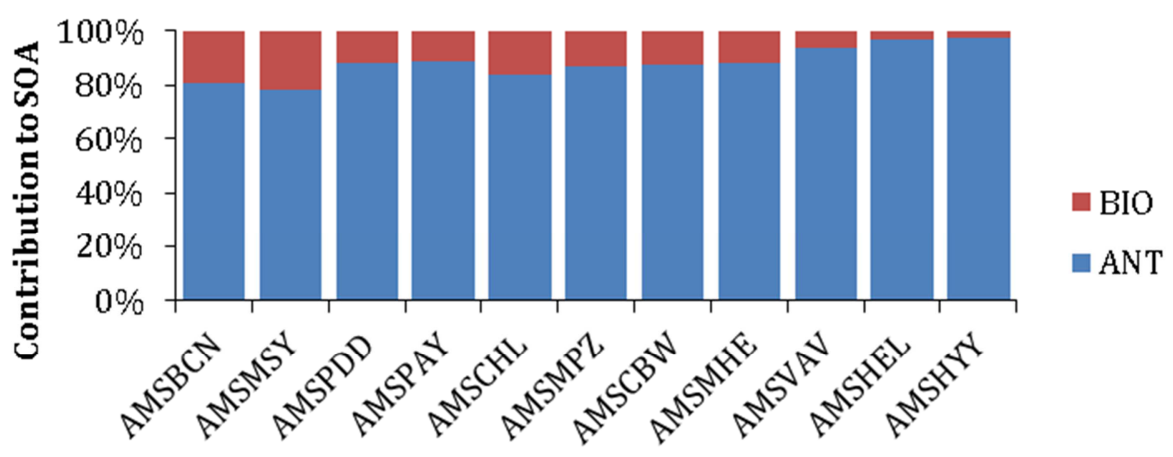

Figure S5. Biogenic and anthropogenic contribution to SOA at stations from south to north retrieved as a difference between the predicted SOA in the reference simulation (including biogenic) and a sensitivity test with no biogenic SOA formation. 


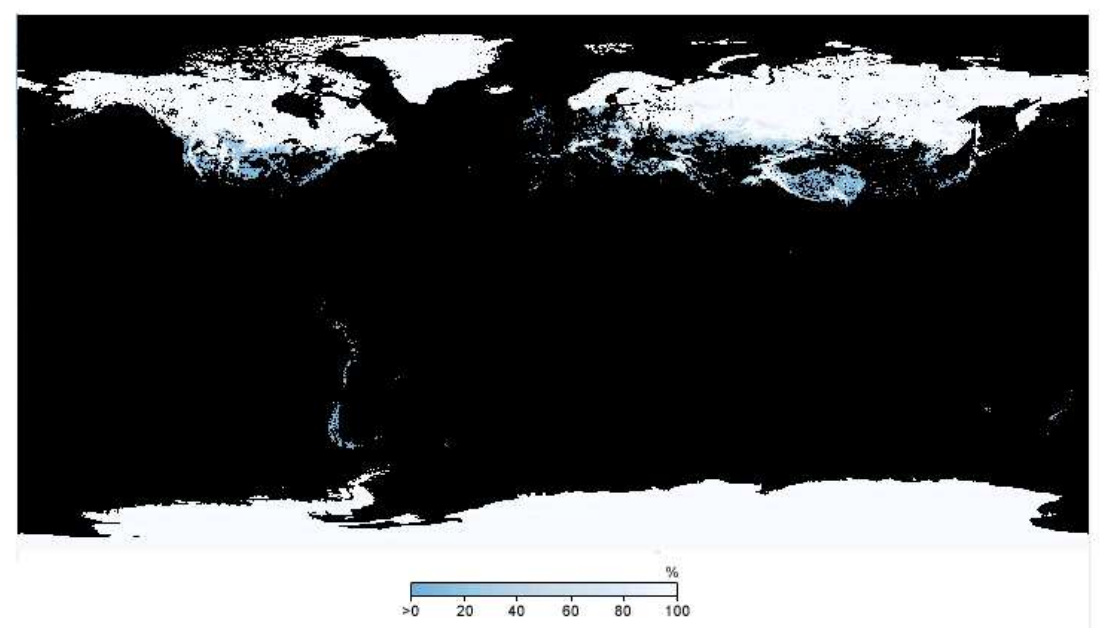

Figure S6. Snow cover for March 2009 as retrieved bythe TERRA/MODIS instrument.
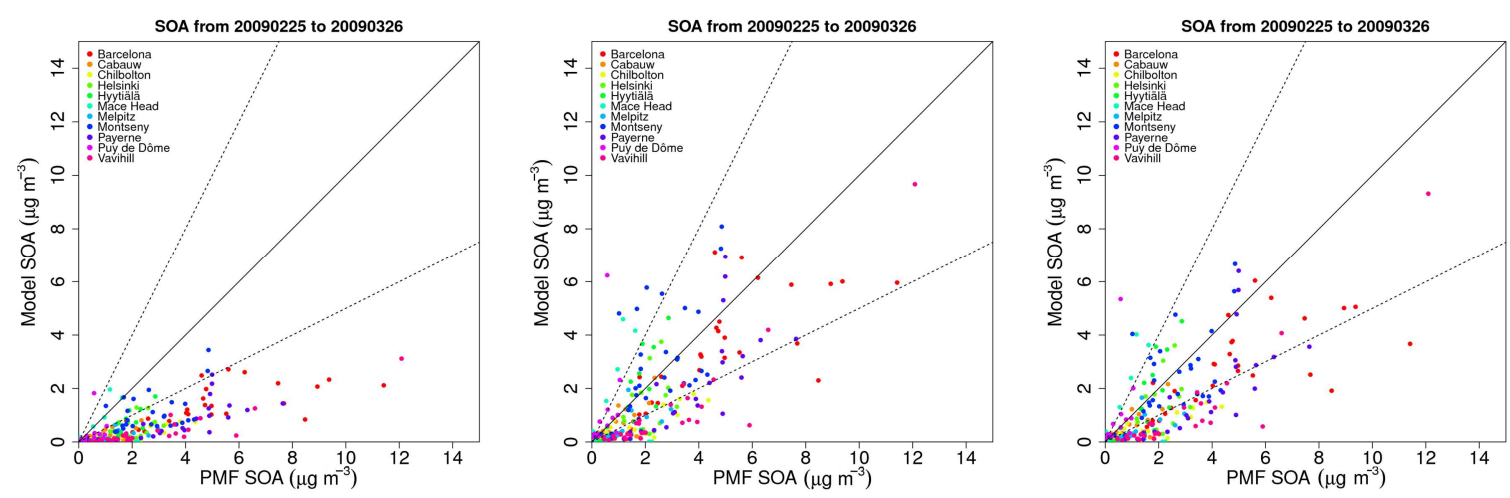

Figure S7. Modelled versus PMF SOA; with VBS_BC (Ciarelli et al., 2016) (left panel), with VBS_BC_NEW (middle panel), and with VBS_BC_NEW but without biogenic SOA (right panel). 